\title{
Synchronization of delayed fluctuating complex networks
}

Cite as: AIP Conference Proceedings 2075, 020005 (2019); https://doi.org/10.1063/1.5091122

Published Online: 26 February 2019

Javier Rodríguez-Laguna, Otti D’Huys, Manuel Jiménez-Martín, Elka Korutcheva, and Wolfgang Kinzel

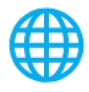

View Online

\section{ARTICLES YOU MAY BE INTERESTED IN}

Universal critical amplitudes in a quantum spherical model: Entropy, internal energy and specific heat

AIP Conference Proceedings 2075, 020010 (2019); https://doi.org/10.1063/1.5091127

Scaling behavior of current-voltage characteristics of $\mathrm{Fe}_{1.02} \mathrm{Se}$ crystal

AIP Conference Proceedings 2075, 020011 (2019); https://doi.org/10.1063/1.5091128

A comparison analysis of the dynamics and stability of Klotho gene network through discrete and hybrid models

AIP Conference Proceedings 2075, 020014 (2019); https://doi.org/10.1063/1.5091131

\section{Conference Proceedings}

\section{Get $30 \%$ off all print proceedings!}

\section{Enter Promotion Code PDF-30 at checkout}




\title{
Synchronization of Delayed Fluctuating Complex Networks
}

\author{
Javier Rodríguez-Laguna ${ }^{1}$, Otti D’Huys ${ }^{2}$, Manuel Jiménez-Martín ${ }^{1}$, \\ Elka Korutcheva ${ }^{1,3, a)}$ and Wolfgang Kinzel ${ }^{4}$ \\ ${ }^{1}$ Departamento de Física Fundamental, UNED, Spain \\ ${ }^{2}$ Department of Mathematics, Aston University, B4 7ET Birmingham, United Kingdom \\ ${ }^{3}$ G. Nadjakov Inst. Solid State Physics, Bulgarian Academy of Sciences, BG-1784, Sofia, Bulgaria \\ ${ }^{4}$ Institute for Theoretical Physics, Würzburg University, Germany \\ a)Corresponding author: elka@ fisfun.uned.es
}

\begin{abstract}
In this communication we present some of our recent results on the synchronization properties of directed delay-coupled networks of a small-world type, whose topology changes with time. Our simulations of a network of non-linear elements show that a random change of topology enhances the stability of a synchronized state, depending on the interplay between different time-scales in the dynamics. The results are analytically explained in the linear limit, where the dynamics is expressed in terms of an effective connectivity matrix. In the limit of fast network fluctuations, this effective connectivity is given by the arithmetic mean of the temporal adjacency matrices. When the coupling topology changes slowly, the effective adjacency matrix is given by the geometric mean. The transition between both regimes is numerically studied for linear network elements.
\end{abstract}

\section{Introduction}

The cooperative behavior of chaotic systems in interaction can lead to partial and global synchronization [1]. Often, the finite traveling speed of information between interacting elements leads to a coupling delay. This coupling delay can induce distinctive patterns of chaos synchronization which have been observed in, for example, neural networks $[2,3]$ and semi-conductor lasers [4]. On the other side, time-varying networks are very common in different contexts such as (and not limited to) social networks or neural networks [6,7]. Synchronization in time-varying networks has been intensively studied in a variety of systems paying attention to the statistical properties of the networks concerning partial or full consensus with applications to control theory [8].

In a previous publication [9] we have incorporated both ingredients: time delay in the interactions and a timevarying topology. We considered an interaction network of coupled chaotic maps (Bernoulli and Logistic) with a single coupling delay, while the connection topology varied with a characteristic time scale among an ensemble of small-world (SW) networks. We found that random fluctuations in the network connectivity may enhance or reduce the stability of a synchronized state, depending on the interplay between the network time-scales. This behavior has been recently theoretically explained in the linear limit [10] by showing that the connectivity matrix can be expressed in terms of an "effective" one, which is given by the arithmetic or geometric average over the different adjacency matrices in the case of fast or slow network fluctuations respectively.

In this paper we present our latest results concerning the synchronization behavior of the above mentioned systems. We first describe our model. Then, we will highlight the theoretical predictions within the fast and slow approximations, comparing with numerical simulations.

\section{Synchronization of delay-coupled chaotic networks}

We study the behavior of $N$ classical units, characterized by a single degree of freedom $u_{i}(t), i \in\{1, \cdots, N\}$ and time $t \in \mathbb{N}$, whose evolution is given by:

10th Jubilee International Conference of the Balkan Physical Union

AIP Conf. Proc. 2075, 020005-1-020005-5; https://doi.org/10.1063/1.5091122

Published by AIP Publishing. 978-0-7354-1803-5/\$30.00 

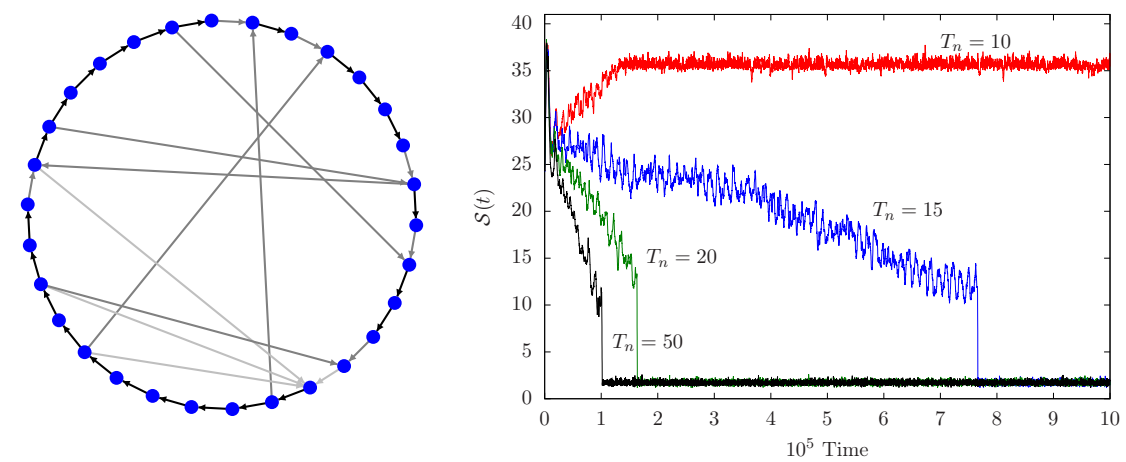

FIGURE 1. Left: A $N=30$ network with a clockwise rotating backbone and $p=0.3$, so the number of shortcuts is $N_{s}=9$. The strength of each link is denoted by its color: black is 1 and grey is $1 / 2$. Right: Synchronization level histories for a fluctuating SW network of Bernoulli maps with different fluctuation times. Parameter values are $N=30, \epsilon=0.6, p=0.5, \tau=100$ for different values of $T_{n}=10,15,20,50$.

$$
u_{i}(t+1)=(1-\epsilon) f\left(u_{i}(t)\right)+\epsilon \sum_{j} A_{i j}(t) f\left(u_{j}(t-\tau)\right) .
$$

Here $\epsilon \in[0,1]$ is a real parameter which measures the strength of the interaction, $\tau$ is the coupling delay and $f:[0,1] \mapsto[0,1]$ is a chaotic map. We consider a Bernoulli map, modelled as $f(x)=a x \bmod 1$ with $a \in \mathbb{R}^{+}$. The coupling topology is given by the network adjacency matrix $A$, which satisfies a stochasticity condition so as to guarantee the existence of a synchronized solution. We analyze a family of Newman-Watts networks [11], keeping a chiral outside ring fixed in order to have a connected network of $N$ sites whose non-zero entries are $A_{i, i+1}$ for $i=1, \ldots N-1$, and $A_{N, 1}$. For every node we establish a directed link to another randomly chosen node with probability $p \in[0,1]$. An example of such a SW network is shown in Figure 1 (left).

\section{Fluctuating networks}

When allowing the network to fluctuate, the connectivity matrix $A$ becomes time-dependent: After a network switching period $T_{n}$ a new matrix $A$ is selected from an ensemble of all row-normalized SW graphs with fixed $N$ and $p$. Thus, all our choices for $A$ guarantee the existence of a synchronized solution, but its stability varies.

In order to characterize the synchronization we have chosen the logarithm of the spatial deviation over the network nodes

$$
\mathcal{S}(t) \equiv-\ln (\sigma(t))
$$

where

$$
\sigma(t) \equiv \sqrt{\frac{1}{N} \sum_{i=1}^{N}\left(u_{i}(t)-\mu(t)\right)^{2}}, \quad \mu(t) \equiv \frac{1}{N} \sum_{i=1}^{N} u_{i}(t)
$$

The synchronization Lyapunov exponent (SLE) $\lambda$, that will be used further, is calculated as the average linear rate at which the synchronization level changes with time $\langle\mathcal{S}(t)\rangle \sim \mathcal{S}_{0}-\lambda t$. In the right panel of Figure 1 we represent the evolution of the synchronization level for a few actual realizations of Eq. (1) of a SW network with $N=30$, $\epsilon=0.6, p=0.5$ and delay $\tau=100$ for different switching times $T_{n}$. The parameters have been chosen so that most static choices of $A$ will lead to de-synchronization [9]. Yet, for relatively fast network switching, $T_{n}=10$, the system synchronizes, while increasing $T_{n}$ and going towards the slow fluctuation limit makes the synchronization unstable, so in actual simulations it de-synchronizes. The time required to de-synchronize decreases with increasing $T_{n}$, an effect that was already reported in Ref. [9]. 


\section{Linearized Model}

In order to understand the physics behind this behavior, we proposed recently a linearized model [10]. This model explains the synchronization effect observed in the fluctuating SW network and the role of the interplay between the different time scales. In the following we briefly summarize the most important results presented in Ref. [10].

Eq. (1) can be linearized around the symmetric solution $u_{1}(t)=u_{2}(t)=\cdots=u_{N}(t) \equiv u_{0}(t)$, yielding an approximate effective equation for small deviations around the synchronization manifold. Defining $x_{i}(t) \equiv u_{i}(t)-u_{0}(t)$ and $\dot{x} \equiv d x / d t$, we have

$$
\dot{\mathbf{x}}(t)=-\lambda_{0} \mathbf{x}+\kappa A(t) \mathbf{x}(t-\tau),
$$

where, for the Bernoulli case, $\lambda_{0}=-\ln |a(1-\epsilon)|$, and $\kappa=a \epsilon$. Notice that $\lambda_{0}^{-1}$ is the time-scale associated with the decay towards synchronization in absence of interaction. There are other two time-scales which are relevant to this problem: the time-delay of the interaction $\tau$, and the network fluctuation time $T_{n}$. The interplay between these three time-scales provides a rich phenomenology.

In some cases, we have been able to show that the synchronization properties of Eq. (4) correspond to a static case with some effective topology, given by a certain effective adjacency matrix $A_{\mathrm{eff}}$, whose properties we will describe.

In the fast network approximation, the network fluctuations are faster than all other time-scales: $T_{n} \ll \lambda_{0}^{-1}$ and $T_{n} \ll \tau$. Therefore, it is very natural to expect that the system will present a similar behavior to a static network with some average topology, as it has been shown in other situations [12]. If we make the basic approximation that

$$
\mathbf{x}\left(t_{0}+T_{n}\right) \approx \mathbf{x}\left(t_{0}\right)+T_{n} \dot{\mathbf{x}}(t) \approx \mathbf{x}\left(t_{0}\right)+T_{n}\left(-\lambda_{0} \mathbf{x}\left(t_{0}\right)+\kappa A_{1} \mathbf{x}\left(t_{0}-\tau\right)\right),
$$

we obtain the following expression at $t=t_{0}+M T_{n}$ and up to first order in $T_{n}$ :

$$
\dot{\mathbf{x}}\left(t_{0}\right) \approx \frac{1}{M T_{n}}\left(\mathbf{x}\left(t_{0}+M T_{n}\right)-\mathbf{x}\left(t_{0}\right)\right) \approx-\lambda_{0} \mathbf{x}\left(t_{0}\right)+\frac{\kappa}{M} \sum_{m=1}^{M} A_{m} \mathbf{x}\left(t_{0}-\tau\right) .
$$

The last expression naturally introduces an "effective" adjacency matrix [10]

$$
A_{\mathrm{eff}}=\frac{1}{M} \sum_{m=1}^{M} A_{m}
$$

By using the above effective adjacency matrix and working within the fast switching approximation, we obtained the decay rate given by the most unstable solution from the Master stability function. Numerical simulations with a network alternating between two non-commuting adjacency matrices $A_{1}$ and $A_{2}$, presented in our previous publication [10], show an excellent agreement with the above exponential decay rate $\lambda$.

In the slow network approximation, the network time $T_{n}$ is similar to the coupling delay $\tau$, and both are larger than the instantaneous decay rate $\lambda_{0}^{-1}$ of the nodes, i.e. $\lambda_{0}^{-1} \ll T_{n} \sim \tau$. The coupling is constant during each delay interval, thus one can perform the analysis by integrating Eq. (4). By using an arbitrary initial conditions $\mathbf{x}_{0}(t), t \in$ $[-\tau, 0]$ and decomposing it into its Fourier components, one can find the evolution of the $n$-th mode during the first delay interval

$$
\mathbf{x}_{1}(t)=\sum_{n} \mathbf{x}_{1 n} e^{i \omega_{n} t}
$$

with

$$
\mathbf{x}_{1 n}=\frac{\kappa}{\lambda_{0}+i \omega_{n}} A_{1} \mathbf{x}_{0 n}
$$

where $\omega_{n}=2 \pi n / \tau$ (see Ref. [10] for details). Repeating this procedure for $M$ alternations of the topology, one finally ends up with an effective adjacency matrix, which in this case is given by the geometric mean of the matrices $A_{1}, A_{2}, \cdots$ along the time evolution

$$
A_{\text {eff }}=\left(\prod_{m=1}^{M} A_{m}\right)^{1 / M}
$$



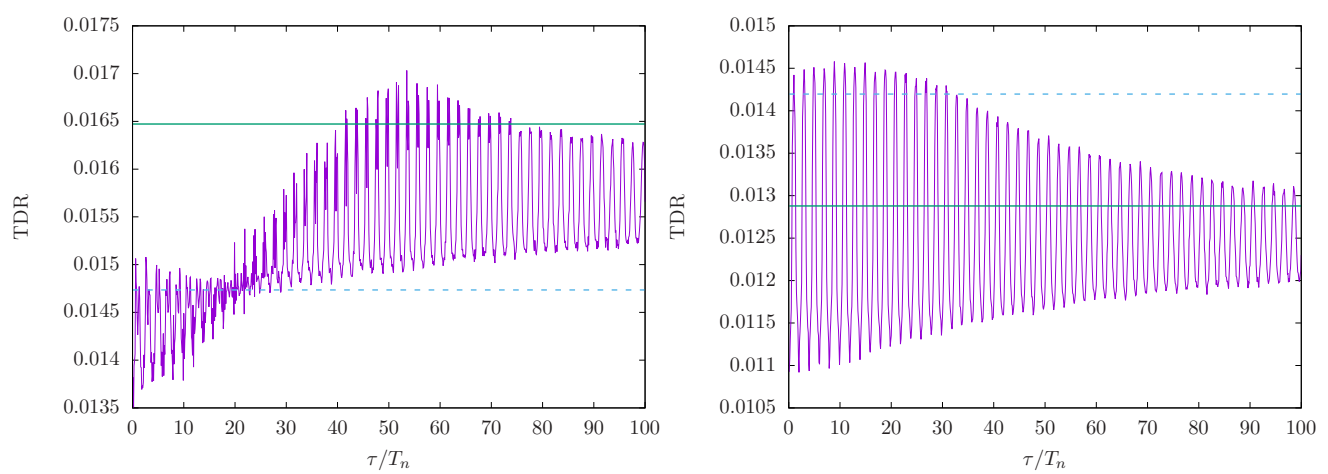

FIGURE 2. TDR for a system composed of $N=9$ nodes, $\tau=100$ and $\lambda_{0}=\kappa=1$. The left and right panels employ different couples of stochastic matrices, randomly selected and non-commuting. Notice the strong parity effect for different ratios $\tau / T_{n}$ reported in Ref. [10]. The horizontal lines correspond to the theoretical predictions: green (continuous) line for the fast switching approximation and blue (discontinuous) for the slow switching one.

Numerical analysis for the decay rates, performed in the case of slow network approximation shows again a very good agreement with the theoretical predictions [10].

\section{Simulations in fluctuating networks}

We can calculate the transverse decay rate (TDR) of the linear system Eq. (4), by numerically integrating the system and estimating the exponential decay rate of the spatial variance of the nodes $\left\{x_{i}(t)\right\}[10]$. For a static adjacency matrix $A$ with second largest eigenvalue $\gamma$ the TDR is expected to behave in the following way $[9,10,13]$ :

$$
\operatorname{TDR}=\frac{1}{\tau} \log \left|\frac{\kappa \gamma}{\lambda_{0}}\right|
$$

Figure 2 shows such TDR as a function of $\tau / T_{n}$ for two different systems with $N=9, \tau=100$ and $\lambda_{0}=\kappa=1$, with a topology alternating between two different sets of two random stochastic matrices. The first salient feature of the two plots is the strong parity oscillation, which is the reason why we chose this representation of the data. This parity effect is also theoretically explained in our previous work [10]. The green (continuous) line provides the predicted TDR associated to fast switching, which is provided by the arithmetic average of the adjacency matrices, while the blue (discontinuous) line provides the TDR associated with the slow switching approximation, which is given by the geometric mean. We can observe a good qualitative agreement between our theory and simulations in Figure 2: for large $\tau / T_{n}$, the TDR curve approaches the fast switching prediction, while for moderate and low $\tau / T_{n}$ the TDR curve approaches the slow switching prediction.

The validity of our predictions regarding the arithmetic and geometrical average of the adjacency matrices extends to the non-linear case of the Bernoulli network given by Eq. (1), as it is shown in our earlier publications [10].

\section{Acknowledgments}

This work was partly supported by the Spanish Government through grant FIS-2015-69617-C2-1-P (J.R.-L.) and the Alexander von Humboldt Foundation within the Renewed research stay program (E.K.). O.D. thanks the European Union's Horizon 2020 research and innovation programme under the Marie Sklodowska-Curie grant agreement No 713694 (MULTIPLY).

\section{REFERENCES}

[1] A. Pikovsky, M. G. Rosenblum, and J. Kurths, Synchronization, A Universal Concept in Nonlinear Sciences, (Cambridge University Press, Cambridge, 2001).

[2] A. Knoblauch et al., Front. Comput. Neurosci. 6, 55 (2012). 
[3] S. Heiligenthal et al., Phys. Rev. Lett. 107, 234102 (2011).

[4] A. Argyris et al., Nature 438, 343 (2005).

[5] I. Kanter, E. Kopelowitz, and W. Kinzel, Phys. Rev. Lett. 101, 084102 (2008).

[6] J. L. Iribarren and E. Moro, Phys. Rev. Lett. 103, 038702 (2009).

[7] M. Tsukada, in: Advances in Cognitive Neurodynamics (V), 689 (2016).

[8] R. Olfati-Saber, American Control Conference, June 8-10, 2371 (2005).

[9] M. Jiménez, J. Rodríguez-Laguna, O. D’Huys, J. de la Rubia, E. Korutcheva, Phys. Rev. E 95, 052210 (2017).

[10] O. D’Huys, J. Rodríguez-Laguna, M. Jiménez, E. Korutcheva and W. Kinzel, ArXiv:1805.03004.

[11] M. Newman and D. Watts, Phys. Lett. A 263, 341 (1999).

[12] D. J. Stilwell, E. M. Bollt, and D. G. Roberson, SIAM Journal on Applied Dynamical Systems 5, 140 (2006).

[13] L. M. Pecora and T. L. Carroll, Phys. Rev. Lett. 80, 2109 (1998). 